\title{
THE ELECTROLYTIC DETERMINATION OF NITRIC ACID WITH A ROTATING ANODE. ${ }^{1}$
}

BY LESLIE HOWARD INGHAM

Receired July 26,1904 .

Luckow first noted that in the electrolysis of dilute nitric acid no ammonia was produced unless copper sulphate was present, whereas, in the presence of the latter, ammonium sulphate was formed while copper was being plated upon the cathode. Vortmann, ${ }^{2}$ in I8go, applied this thought to the quantitative estimation of nitric acid. When all the copper was depasited, the residual liquid was evaporated to a small bulk and, excess of alkali having been added, the ammonia was distilled off into an excess of standard acid and this excess was then titrated back with standard alkali.

Easton, in $1903,{ }^{3}$ studied the procedure of Vortmann and published the conditions which he found to be satisfactory. He distilled off the ammonia and determined it in the usual manner.

The present study of the method was undertaken primarily with a view of ascertaining whether the time of determination could be shortened. Agitation of the electrolyte being known to promote the rapid deposition of metals, it was thought that it might also favor the rapid reduction of nitrates. Another mode of shortening the time would be the omission of the distillation of the ammonia. A stiggestion of Vortmann, ${ }^{2}$ quated by Easton, but not used by him, was to acidulate the solution of the nitrate with a known amount of standardized acid and add a solution of copper sulphate containing a definite amount of sulphuric acid. After the reduction of the nitrate this acid would be partly neutralized by the ammonia which had been formed and the excess of acid could be then directly titrated by standard alkali.

This was the course adopted by the writer. The apparatus employed in the reduction was that used in the rapid determination of zinc.

In each experiment the desired quantity of potassium nitrate

1 From the author's thesis for the Ph.D. degree.

2 Ber. d. chem. Ges., 1890 , p. 2798.

8 This Journal, 25 , 1042. 
was weighed off and dissolved in a small amount of water in a clean platinum dish; the necessary amount of copper sulphate was then pipetted from the stock solution and a measured amount of standard sulphuric acid was added, sufficient to make the electrical resistance low and to insure the solution remaining quite strongly acid during the reduction of the nitrate.

This solution was then diluted to about $125 \mathrm{cc}$. and electrolyzed with a current of from 4 to 5 amperes and about ro volts. The exact conditons are stated for each experiment in the tabular exhibit. During the electrolysis the copper was deposited upon the cathode and its equivalent of sulphuric acid was liberated and added to the acid already present, whereby the conductivity was increased and the voltage fell. As the nitric acid was gradually reduced to ammonia the free acid was neutralized and if the current was maintained constant by the rheostat, the voltage gradually rose for about twenty-eight minutes and then became stationary, thereby indicating the end of the reduction. This rise was usually from 5 to 7 volts. The voltages given in the table are those read at the outset of each experiment, to which the above must be added to obtain the final voltage.

The motor having been stopped, the liquid in the dish was siphoned off into a beaker and replaced by distilled water while the current continued; the dish, anode and cover glasses were well washed, the electrical circuit interrupted, and the washings added to the liquid in the beaker. It being unnecessary to weigh the deposited copper, the platinum dish was merely rinsed with nitric acid and washed under the faucet, when it was ready for use again.

The contents of the beaker were rapidly neutralized in the presence of litmus or methyl orange by the standard ammonia solution. The indicators named were found to give identical results. Let it be noted that in the reduction I molecule of potassium nitrate gives rise to a molecule of potassium hydroxide and one of ammonia; hence two equivalents of alkali are produced from one equivalent of nitrate, and allowance must be made for this by halving the results oblained by titration. The use of a 0.5 -gram sample for analysis just offsets this. The calculation of the standard ammonia solution to its equivalent of $\mathrm{N} / 5$ sodium carbonate solution and thence to nitrogen is obvious.

A number of experments we:e conducted to ascertain the time, 
the amount of copper sulphate, the current strength and speed best suited for the quantitative reduction of nitrates.

(a) Time.-The first ten experiments were made with reference to the time of reduction. Using $25 \mathrm{cc}$. of copper sulphate solution, $25 \mathrm{cc}$. of acid solution and 0.5 gram of nitrate, 5 amperes gave 5.63 per cent., 9.83 per cent., 9.91 per cent. and II.26 per cent. of nitrogen respectively in ten, fifteen, fifteen and twenty minutes, the theoretical percentage of nitrogen in potassium nitrate oeing I 3.86 .

By increasing the time, 4 amperes gave I 3.64 per cent. in twenty five minutes and $\mathrm{r} 3.83$ per cent. in thirty minutes.

(b) Amount of Copper Sulphate.-The above results were obained with $25 \mathrm{cc}$. of copper sulphate. Two experiments with $30 \mathrm{cc}$. gave 8.79 per cent. in twenty minutes and 12.96 per cent. on thirty minutes, showing that the increased amount of copper is not an advantage. Two experiments with but $15 \mathrm{cc}$. of copper sulphate solution and $30 \mathrm{cc}$. of standard acid resulted in a reiuction of II.93 per cent. and 13.55 per cent. in twenty and thirty minutes respectively. Increasing the amount of acid to $50 \mathrm{cc}$. with the same amount of copper gave better results, viz., I3.IO per cent. and 13.83 per cent. in twenty and thirty minutes respectively.

(c) Strength of Current.-An experiment with 5 amperes gave $3.3^{8}$ per cent. nitrogen in twenty-five minutes, while 6 amperes gave only 13.19 in twenty minutes. From this it appears that amperes is sufficient current, since that will yield complete reuction in thirty minutes and more current will not do the work in less time.

(d) Speed.-Two experiments with the speed of rotation of are anode increased to about 560 revolutions per minute gave 2.9I per cent. and I3.I9 per cent. in twenty and thirty minutes respectively; the voltage needed was 40 , since the contact between are anode and the liquid was poor at this velocity. So much eat was produced that the liquid boiled freely, but no advantage was found in increased speed.

The results and detailed conditions of this work are found in le subjoined table. 


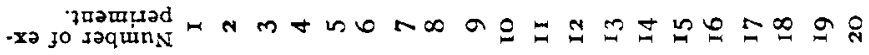
นpanoj

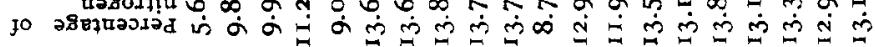

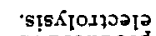

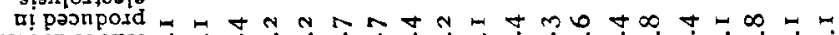

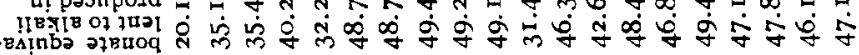

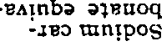

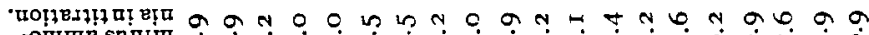

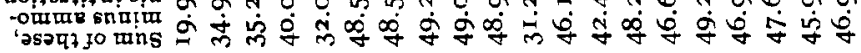

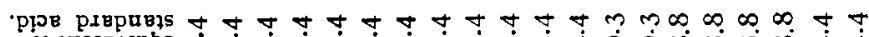

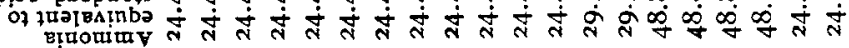

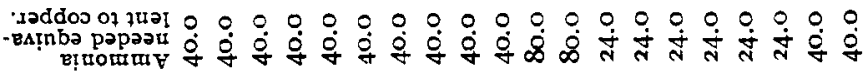

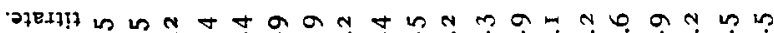

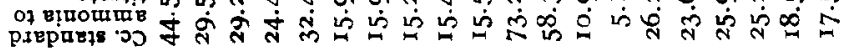

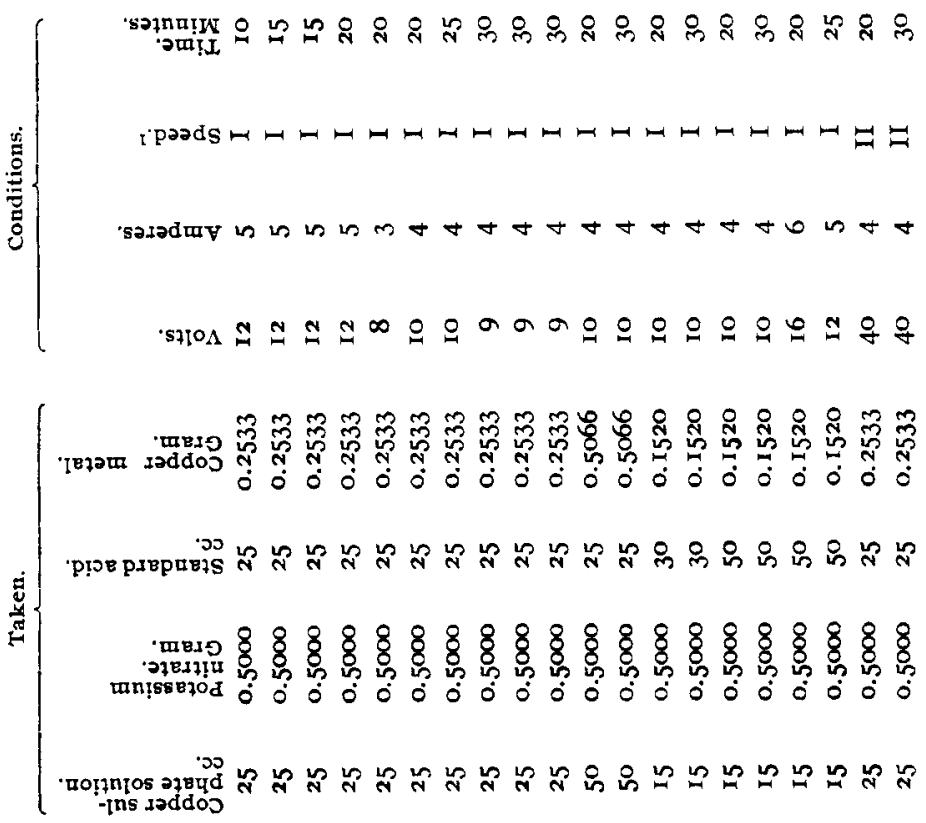


The above results appear to indicate that the conditions of Experiment 8 are to be preferred. To confirm this a series of ten determinations were made in accordance with those conditions, namely, $25 \mathrm{cc}$. of copper sulphate solution, representing 0.2533 gram of metallic copper, $25 \mathrm{cc}$. of the standard sulphuric acid, .5 gram of patassium nitrate, 4 amperes, Io volts at the outset, $r$ I 7 volts at the end of reduction, slowest speed and thirty minutes. The dish was not warmed at the outset of the experiment, or was external heat applied during electrolysis, although the liquid was considerably heated by the current, the final temperature being about $65^{\circ} \mathrm{C}$. This continuous series was made in a single afternoon and no results were rejected; consequently they may be taken as representing the probable error of the method.

The following are the percentages of nitrogen found, the theoretical value being I 3.86 :

$\begin{array}{ccccc}\text { Per cent. } & \text { Per cent. } & \text { Per cent. } & \text { Per cent. } & \text { Per cent. } \\ \text { I3.8I } & \text { I3.79 } & \text { I3.83 } & \text { I3.83 } & \text { I3.94 } \\ \text { I3.86 } & \text { I3.92 } & \text { I3.92 } & \text { I3.86 } & \text { I3.89 } \\ & \text { Mean of the series of ten, } & \text { I3.865. } & \end{array}$

This method for nitrates compares quite favorably with other methods in point of accuracy. Its advantages in simplicity and speed appear to the writer to be worthy of careful consideration, as a complete determination of the nitric acid content of an alkali litrate may be made in thirty-five minutes from the time of beighing off the sample.

The applicability of the method to other nitrates than that of potassium will be studied.

CONTRIBUTION FROM THE JOFN HARRISON LABORATORY OF CHEMISTRY, No. 85.]

\section{DETERMINATION AND SEPARATION OF GOLD IN THE ELECTROLYTIC WAY. ${ }^{1}$}

\section{By Saraf P. Miller.}

Received July 26, 1904 .

The purpose of this investigation was to ascertain the conditions under which gold could be satisfactorily deposited from its soluon:

(a) in potassium cyanide;

(b) in the presence of phosphoric acid;

1 From the author's thesis for the Ph.D. degree. 